Supporting Material for the paper:

PARTial POly-(GLUTAMIC ACID) $\leftrightarrow$ POLY-(ASPARTIC ACID) EXCHANGE IN LAYER-BY-LAYER POLYELECTROLYTE FILMS. STRUCTURAL ALTERATIONS IN THE THREE-COMPONENT

\title{
ARCHITECTURES
}

Ana-Maria Pilbat, ${ }^{\dagger}$ Vincent Ball, ${ }^{\S}$ Pierre Schaaf, ${ }^{\ddagger}$ Jean-Claude Voegel, ${ }^{\S}$ and Balázs

\section{Szalontai $*^{\dagger}$}

Institute of Biophysics, Biological Research Center of the Hungarian Academy of Sciences, Szeged, Temesvári krt. 62, H-6701 P.O.B. 521, Hungary, Institut Charles Shadron, Unité Propre 22, Centre National de la Recherche Scientifique, 6 rue Boussingault, 67083 Strasbourg Cedex, France, and Faculté de Chirurgie Dentaire, UMR 595, Institut National de la Santé et de la Recherche Médicale, 11, rue Humann, 67085 Strasbourg Cedex, France

The structures of the component amino acids and the polyelectrolyte multilayers built from them.

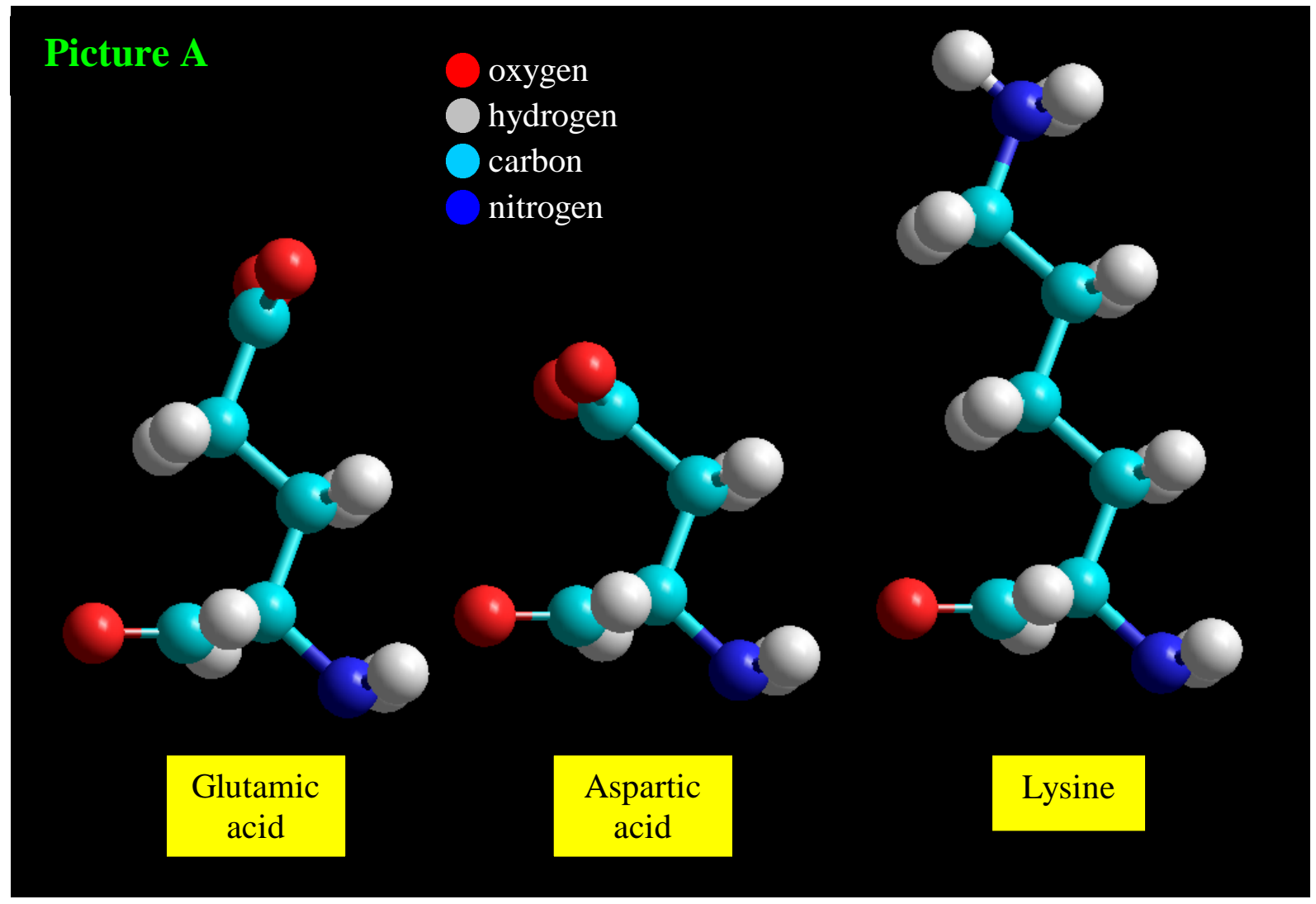


Picture A shows the structures of the three amino acids used in the experiments. Picture B shows the complex of PGA with PLL for an idealistic case, and only for three repeating units. Nevertheless, it can be seen that the $\beta$-structure of the complex, we postulated from the infrared spectra are maintained by the electrostatic interaction between the charged side chains. Those are the red (oxygen) and blue (nitrogen) atoms. The backbones of both polyamino acids have a $\beta$ sheet conformation, indicated by the green ribbon. In Picture $\mathbf{C}$, the same PGA/PLL complex is shown from another projection. The alternating amino acid side chains on the other side of the $\beta$ sheets form two individual 'lines' of positively and negatively charged amino acid side chains, capable of binding other PGA/PLL complexes as the construction of the polyelectrolyte film goes on. One has to emphasise, that these are not precisely calculated structures only geometry optimisation was carried out, but they can help to imagine how the structure of these complexes may look like. In the case of the large $30-50 \mathrm{kDa}$ polyamino acids, at the beginning probably only small sections of the backbones are in such a perfectly matching situation.

In Picture D, the cross section of a PAA/PLL 'chain is given. It can be seen, that, indeed, from such chains multilayered structures can be built, since there are well separated positively and negatively charged 'edges' of the chain. In Picture E, PGA/PLL is shown from the same projection. Comparing the two pictures, one may have a clue why the incorporation of PAA into PGA/PLL film did not caused major problems, and why were extra structural changes upon the incorporation of PGA into PAA/PLL multilayers. Due to the shorter side chain in aspartic acid, the structure of the PAA/PLL film is more dens (imagine piling up the structure in Picture D, by rotating each second layer by $180^{\circ}$ ) than that of the PGA/PLL film (Picture E).

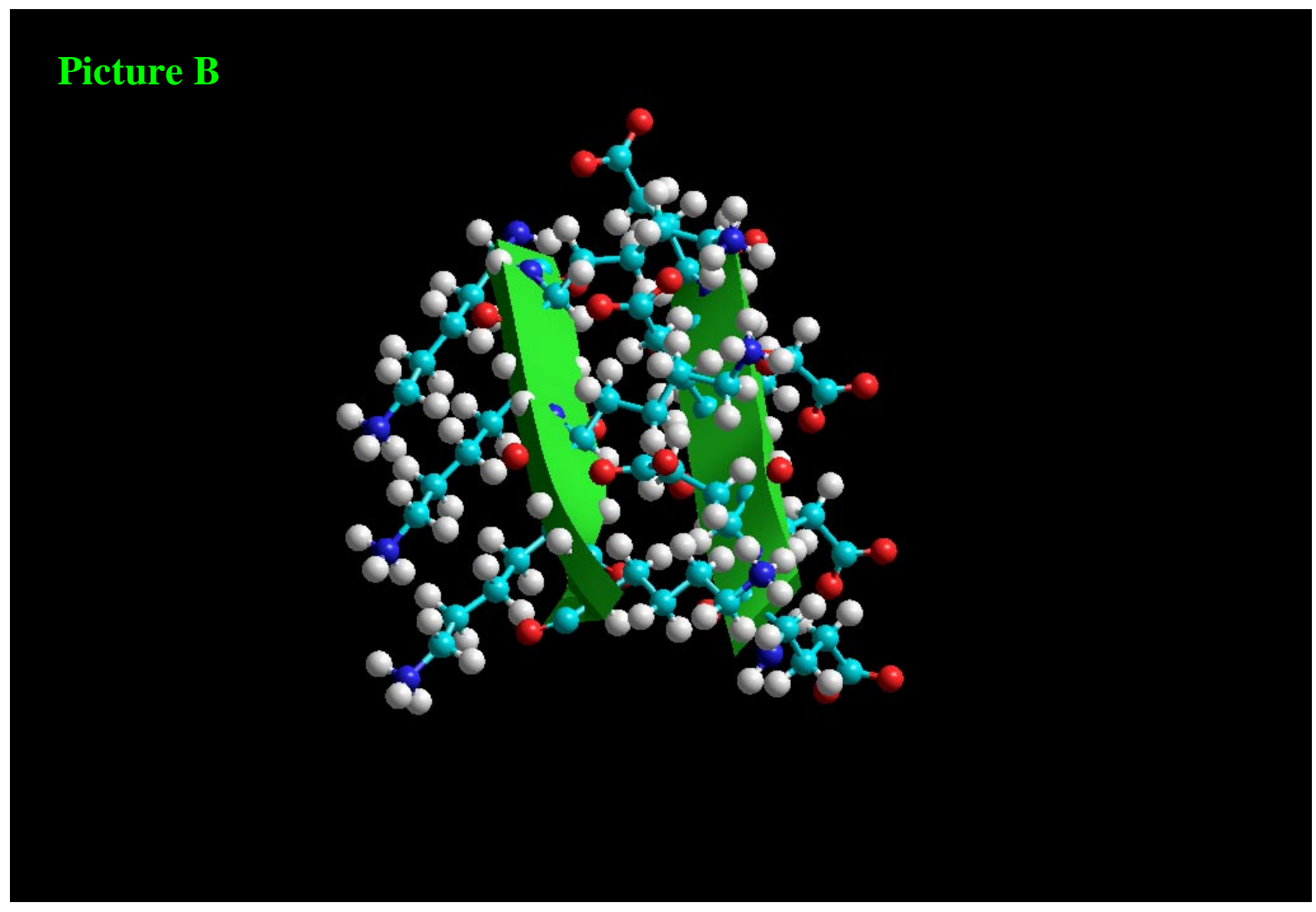



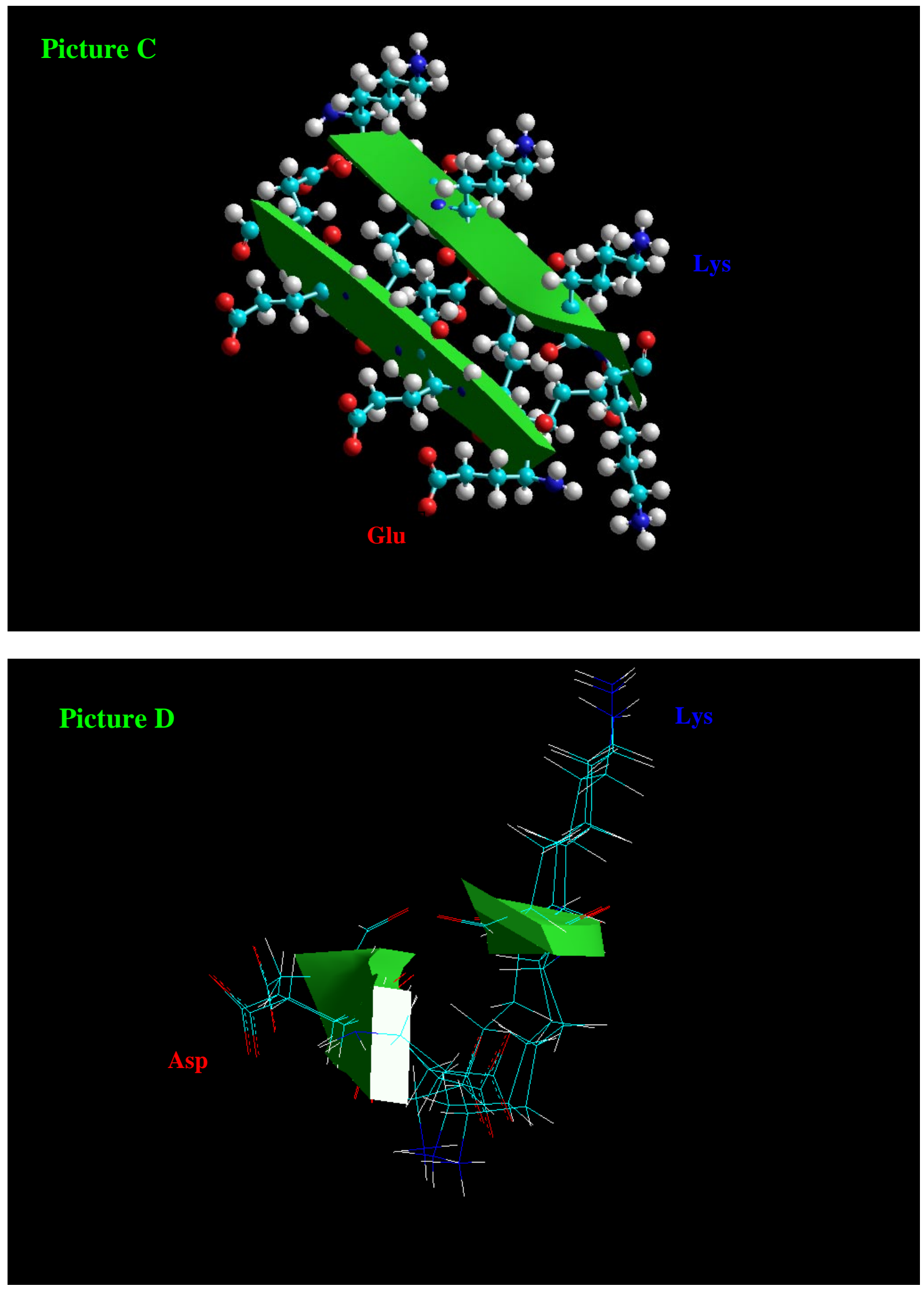


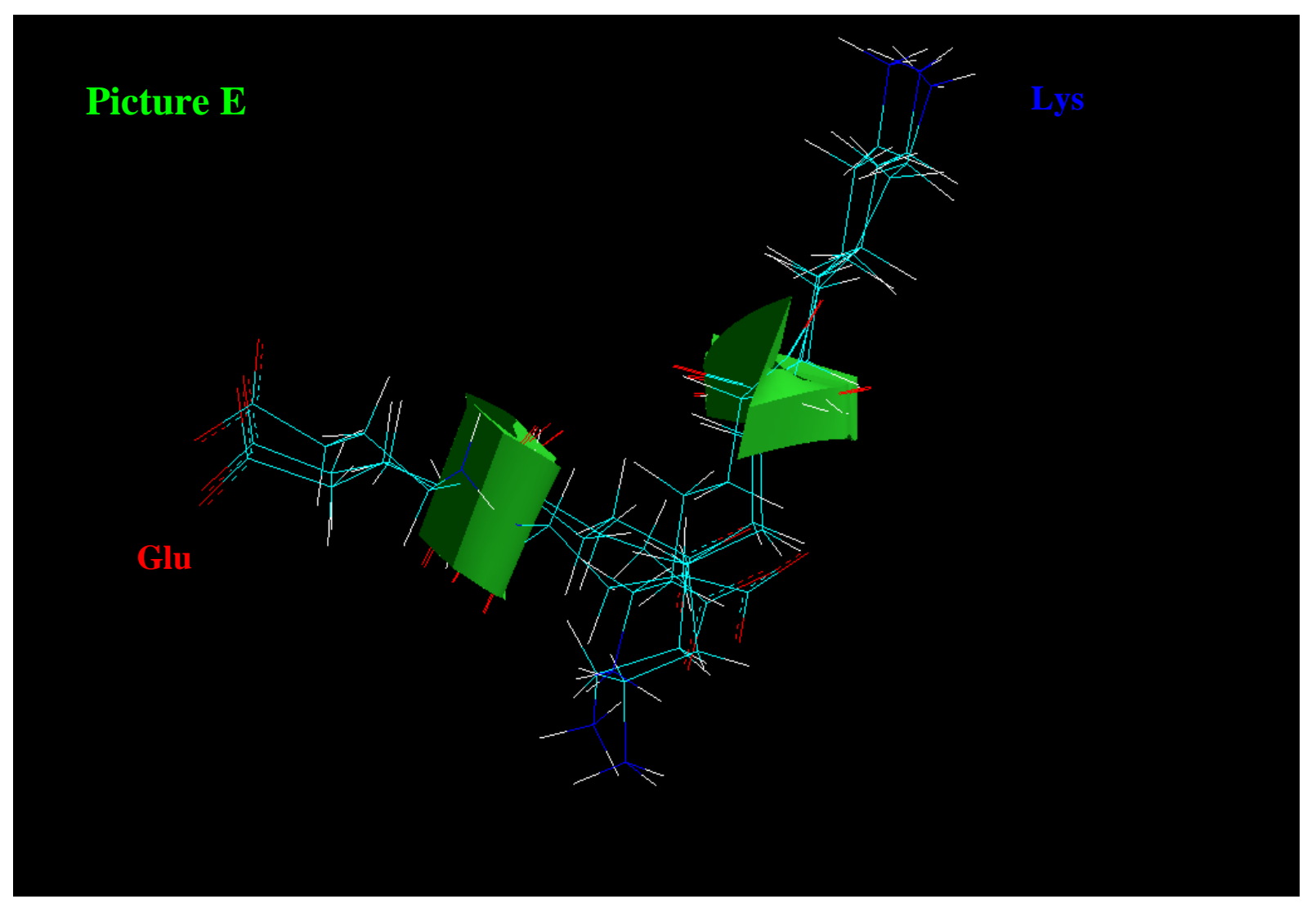

The use of the $4^{\text {th }}$ derivate to determine components in the infrared spectra

To prove the credibility of Fourier smoothing and the following determination of component band frequencies from the second and the fourth derivates, we show three examples: A strong, an intermediate, and a slight Fourier smoothing and the derivates obtained from them.

Note, that the residuals between the measured and the Fourier-smoothed spectra do not show any major distortion as evidenced by the straight baseline of the residuals. The decreasing smoothing has an effect on the features in the amide I region of the residuals. With decreasing smoothing, these features, which might be related to secondary structure elements, become less and less structured.

Note, that the noise of the fourth derivate even in the case of slightest smoothing remains at an acceptable level. To judge this, regard the protein-free $1700-1800 \mathrm{~cm}^{-1}$ region.

It can be seen, that with decreasing smoothing, the derivates become more and more structured. Components, which were not resolved at strong smoothing or were indicated only by a shoulder, can be nicely distinguished. 


\section{HSA demo a - strongest smoothing}

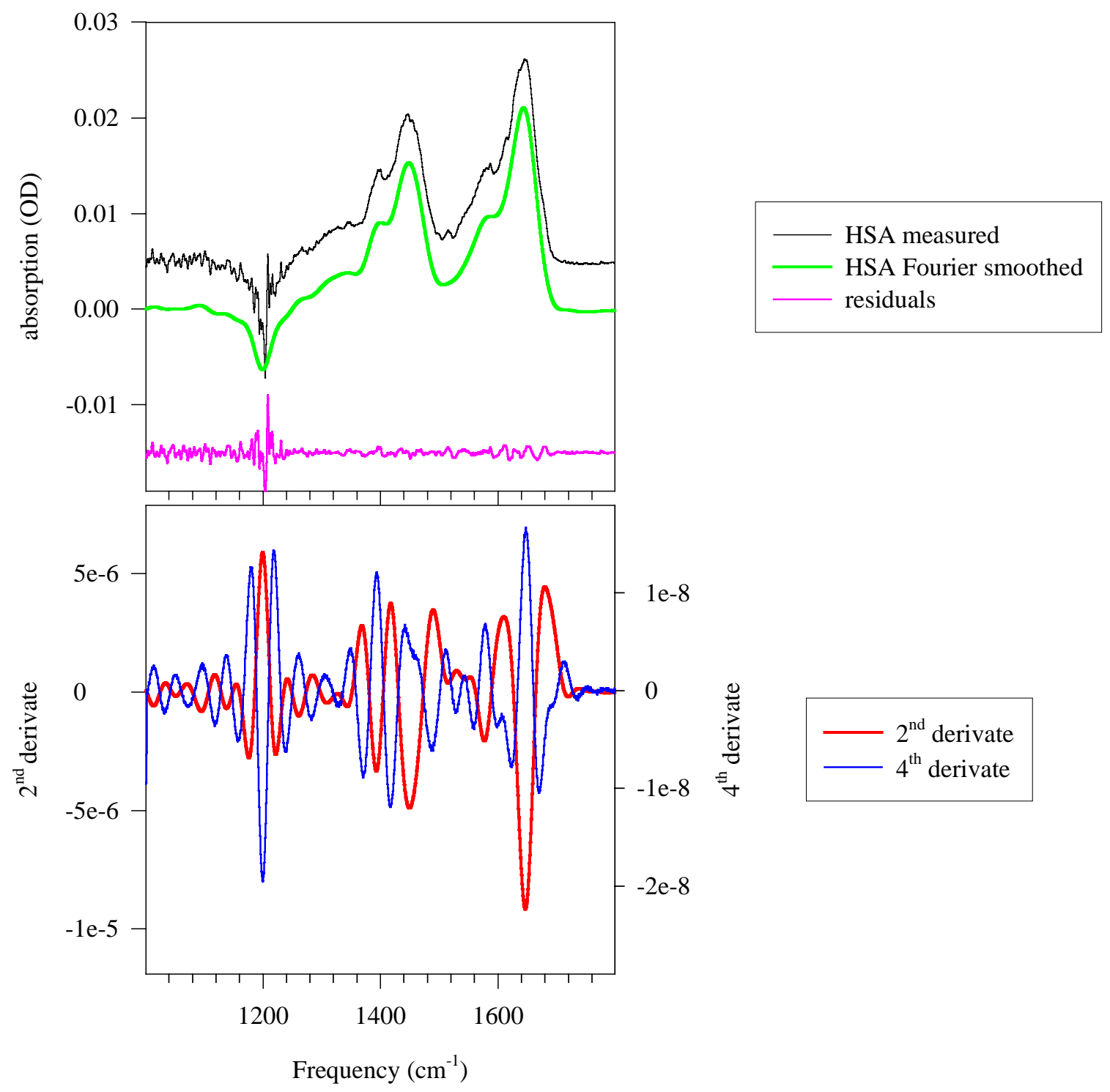




\section{HSAdemo b - intermediate smoothing}
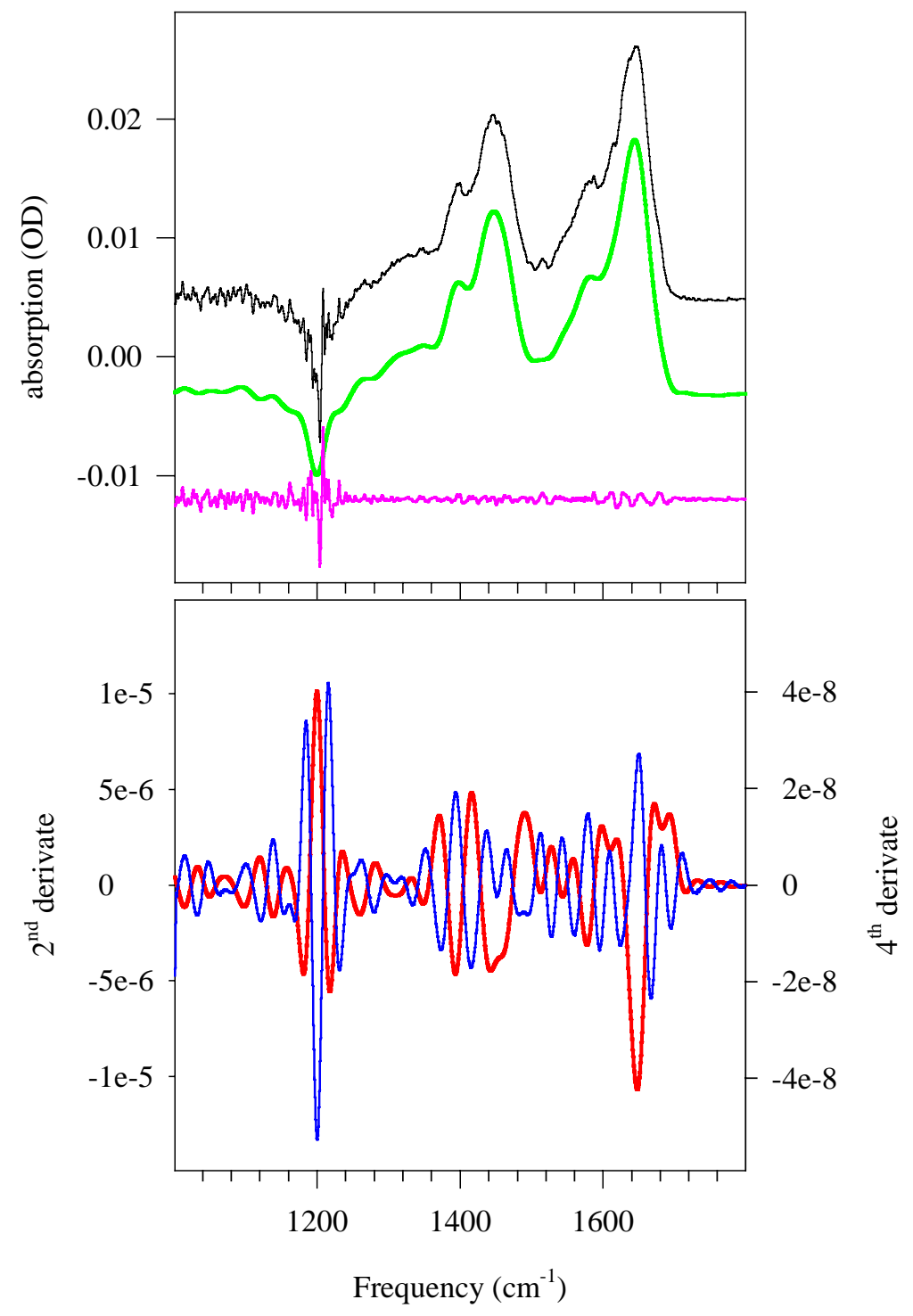

HSA measured spectrum HSA Fourier smoothed residuals

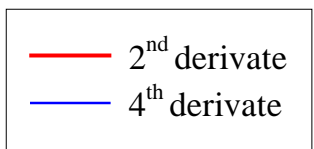


hsademo c - slightest smoothing

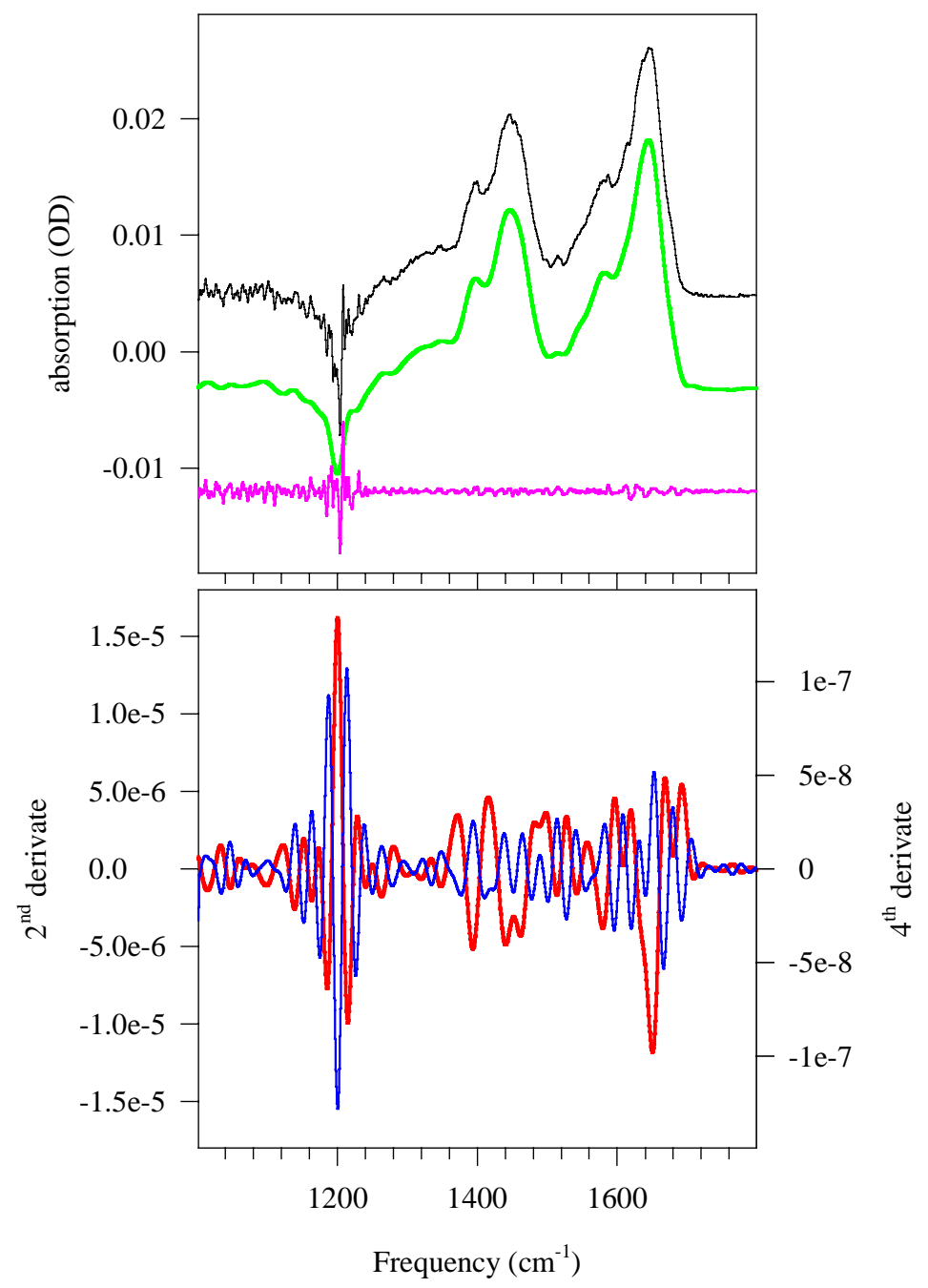

HSA measured spectrum

HSA Fourier smoothed spectrum residuals 


\section{Transient absorption changes in the infrared spectra of (PGA-PLL) 5 -PGA+PAA and (PAA- PLL) 5 -PAA+PGA interactions}

Figure 3 shows the infrared difference spectra induced by the interaction of PAA with a PEI(PGA/PLL) 5 -PGA film (PAA ${ }_{\mathrm{PGA}}$ spectrum in Figure 3A), and that of PGA with a PEI(PAA/PLL) $)_{5}$-PAA film (PGAPAA spectrum in Figure 3B).

These difference spectra (open symbols) were evaluated by the following logic: The difference spectra observed due to entering and leaving polyanions is the net result of the exchange. In the film, PGA and PAA form complexes with PLL. For leaving PGA or PAA, such a complex has to brake up; for an incorporating PAA or PGA, such a complex has to be formed. We know that the infrared spectra of PGA, or PAA are different from that of the (PGA/PLL), or (PAA/PLL) complexes.

Therefore, we have tried to fit these difference spectra with the linear combination of pure PAA and PGA spectra. The spectra of the first PAA and PGA layers on PEI (PAAPEI and PGA PEI, shown in Figure 4B) were used for this purpose. The good fit obtained for PAA $A_{\mathrm{PGA}}$ (Figure 4A) by using the linear combination of PAAPEI and PGAPEI indicates that neither PAA nor PGA was suffering structural changes upon the PGA $\rightarrow$ PAA exchange. A rough estimation, based on the normalized amide I band intensities of the PGA $\mathrm{PEI}_{\text {and }}$ PAAPEI spectra used for the fit gave for one leaving glutamic acid unit seven incorporating aspartic acid units during the exchange. (This estimation includes the approximation that the extinction coefficients of PGA and PAA in the amide I region are the same.) 

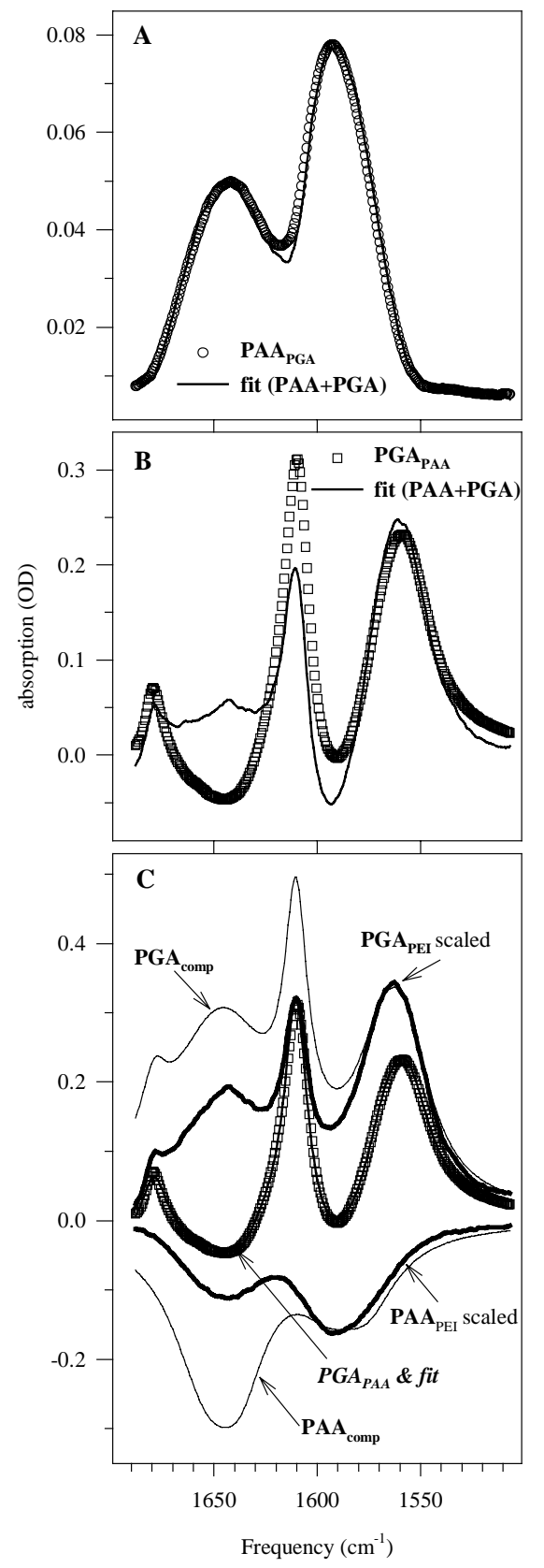

Figure 4. Difference FTIR-ATR spectrum due to adding of PAA to a PEI-(PGA/PLL) $)_{5}$-PGA film (PAA ${ }_{\mathrm{PGA}}$, panel A); adding of PGA to PEI-(PAA/PLL) 5 -PAA film ( $\mathrm{PGA}_{\mathrm{PAA}}$, panel B). Open symbols indicate measured spectra, the solid line is the result of a fit obtained by the linear combination of pure PAA and PGA spectra adsorbed onto a PEI single layer $\left(\mathrm{PAA}_{\mathrm{PEI}}\right.$, and $\mathrm{PGA}_{\mathrm{PEI}}$ ), respectively. $\mathrm{PAA}_{\mathrm{PEI}}$ and $\mathrm{PGA}_{\mathrm{PEI}}$ are shown with scaled amplitudes as obtained for PGA $_{\mathrm{PAA}}$ (panel C). $\mathrm{PGA}_{\text {comp }}$ and $\mathrm{PAA}_{\text {comp }}$ are calculated spectra obtained by summing up all PGA- and PAArelated components, respectively, used in the multi-component fit of $\mathrm{PGA}_{\mathrm{PAA}}$ (the result of the multicomponent fit is the solid line within the open symbols, panel $\mathbf{C}$ ). The components used in the multicomponent fit are listed in Table I. For details, see the text. 


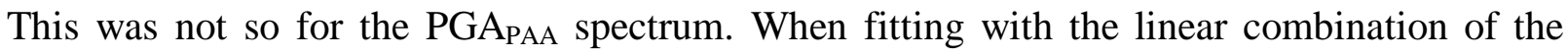
same PAAPEI and PGAPEI spectra as above, there remained large differences between the measured and fitted spectrum, especially in the amide I region (Figure 4B). The same rough estimation as used above gave three leaving aspartic and four incorporating glutamic units for this poor fit. The spectra of PAAPEI and PGA $A_{P E I}$ used in this fit (and also for fitting PAAPGA

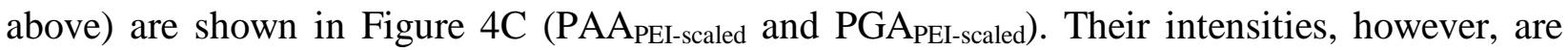
scaled, as required by the fit of PGA $_{\mathrm{PAA}}$ in Figure 4B. The discrepancy between the measured and the fitted spectrum in the amide I region of $\mathrm{PGA}_{\mathrm{PAA}}$ indicates that there must be structural rearrangements in the film upon the PAA $\rightarrow$ PGA exchange.

To reveal the changes in the secondary structure of the PEI-(PAA/PLL) $)_{5}[\mathrm{PAA}+P G A]$ film, we have tried the following approach: At first, we fitted separately both PAA $A_{P E I}$ and PGAPEI with a set of Lorentzian components. PAA needed four, PGA five components for fitting the 1500-1690 $\mathrm{cm}^{-1}$ region. (The components are listed in Table I in the main paper, the fitted spectra are not shown.)

Then, PGA ${ }_{\text {PAA }}$ was fitted with all the nine components of PAA and PGA, but the frequencies and the widths of the components were fixed, only the intensities could be changed during the fit. In this case, excellent fit was obtained (see the curve in the interior of the PGA (open squares in Figure 4C). All PGA components had positive intensities, and all PAA components had negative intensities in agreement with a PAA $\rightarrow$ PGA exchange.

In Figure 4C, $\mathrm{PGA}_{\text {comp }}$ and PAA $\mathrm{A}_{\text {comp }}$ are calculated spectra. They were obtained by summing up all the PGA- and PAA-related component bands, respectively, which were obtained in the fit of the PGA PAA $_{\text {spectrum. PGA }}$ PEI-scaled and PAAPEI-scaled spectra are the scaled components of the poor fit (shown in Figure 4B) obtained for PGAPAA by the linear combination of PGAPEI and 


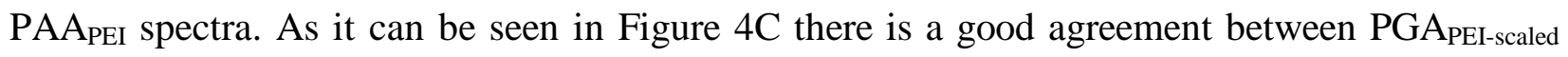
and $\mathrm{PGA}_{\text {comp }}$ spectrum in the $\mathrm{COO}^{-}$stretching region. In the amide I region, however, the intensities of the 1610 and $1647 \mathrm{~cm}^{-1}$ bands are higher in the $\mathrm{PGA}_{\text {comp }}$ spectrum than in the PGA PEI-scaled spectrum.

PAA $A_{\text {PEI-scaled }}$ is also similar to $\mathrm{PAA}_{\text {comp }}$ in the $\mathrm{COO}^{-}$region. Note, that these spectra have negative amplitudes, since PAA left the polyelectrolyte film upon the exchange with PGA. In the amide I region, however, according to the $\mathrm{PAA}_{\text {comp }}$ spectrum, more PAA-related structure is missing than it could be expected on the basis of the PAAPEI-scaled component. This may mean that the intruding PGA molecules, when they finally incorporate into the existing PAA/PLL architecture force some of the PAA molecules to adopt 'PGA-like' conformations. This more negative amide I region here is the source of the surplus of the PGA-related components with extra intensities in the $\mathrm{PGA}_{\text {scaled }}$ spectrum shown in the upper part of Figure $4 \mathrm{C}$. 
Changes in the infrared spectra of pure PAA/PLL and PGA/PLL films upon their layer-bylayer construction:

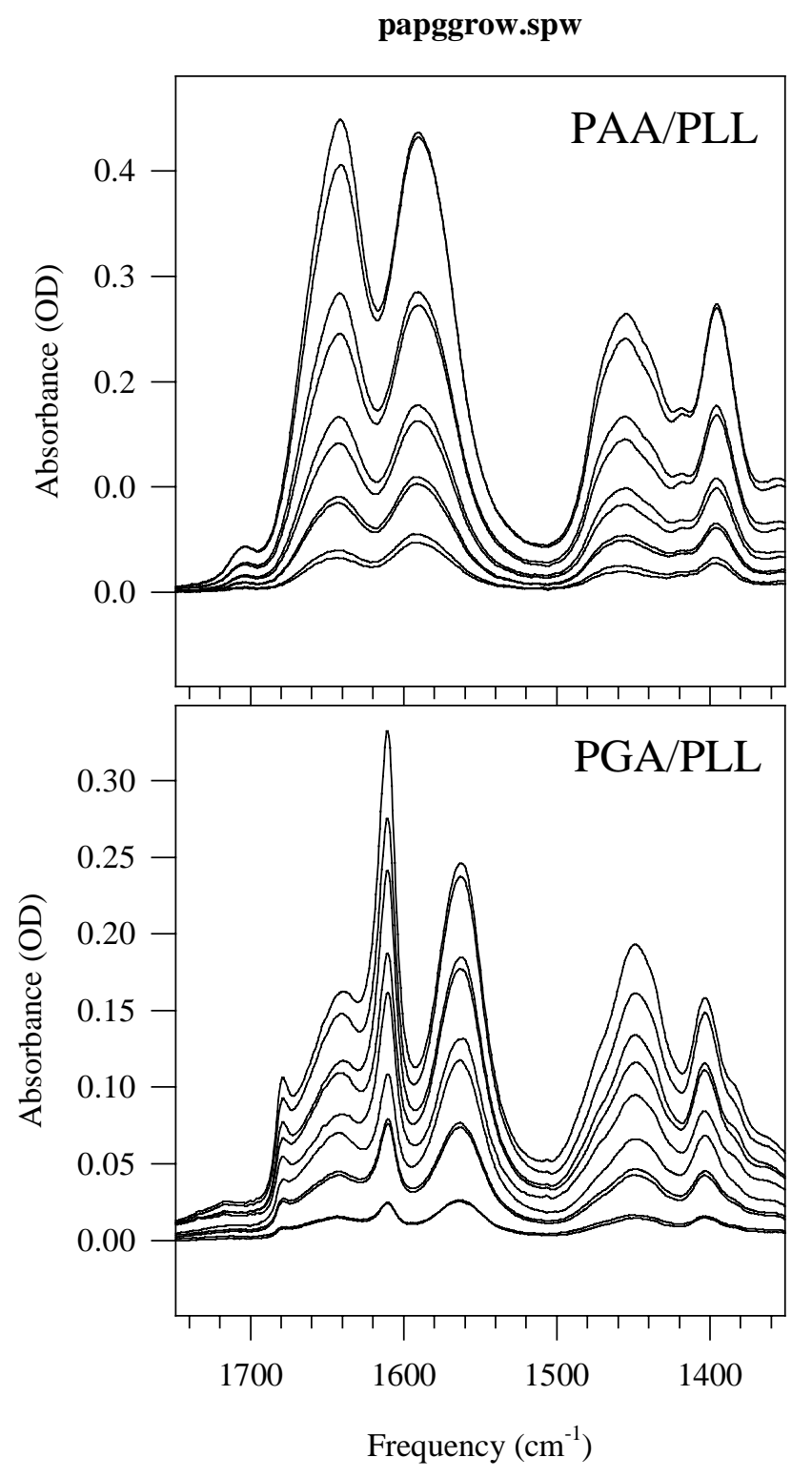

The figure shows the five double layers starting from the bottom to the top. Note, that with increasing number of the layers the 'ordering' effect of PLL is also increasing $\left(2^{\text {nd }}, 4^{\text {th }}, 6^{\text {th }}, 8^{\text {th }}\right.$, $10^{\text {th }}$ spectra from the bottom). I mean by 'ordering' that those features, which are related to the characteristic secondary structure of the given film increase faster than the polyelectrolyte-related bands. The increase of the band at around $1638 \mathrm{~cm}^{-1}$ in PAA/PLL is faster than the increase of the PAA COO band at $1594 \mathrm{~cm}^{-1}$; the increase of the $1610 \mathrm{~cm}^{-1}$ band in the PGA/PLL film is faster than that of the $1567 \mathrm{~cm}^{-1}$ PGA $\mathrm{COO}^{-}$band.

That was the reason why we have chosen the (PGA-PLL) $)_{5}$ and (PAA-PLL) ${ }_{5}$ spectra to fit the mixed (PGA-PLL) 5 -PGA+PAA-PLL or (PAA-PLL)-PAA+PGA-PLL films. 